\title{
Investigating Earth shadowing effect with DAMA/LIBRA-phase1
}

\author{
R. Bernabei ${ }^{1,2, a}$, P. Belli ${ }^{1,2}$, S. d'Angelo ${ }^{1,2}$, A. Di Marco ${ }^{1,2}$, F. Montecchia ${ }^{1,2,3}$, A. d'Angelo ${ }^{4,5}$, A. Incicchitti ${ }^{4,5}$, \\ F. Cappella $^{6}$, V. Caracciolo $^{6}$, R. Cerulli ${ }^{6}$, C. J. Dai ${ }^{7}$, H. L. He $^{7}$, H. H. Kuang ${ }^{7}$ X. H. Ma ${ }^{7}$ X. D. Sheng ${ }^{7}$, \\ R. G. Wang ${ }^{7}$, Z. P. Ye $\mathbf{Y}^{7,8}$ \\ ${ }^{1}$ Dip. di Fisica, Università di Roma “Tor Vergata”, 00133 Rome, Italy \\ 2 INFN, sez. Roma "Tor Vergata", 00133 Rome, Italy \\ ${ }^{3}$ Dip. di Ingegneria Civile e Ingegneria Informatica, Università di Roma "Tor Vergata”, 00133 Rome, Italy \\ ${ }^{4}$ Dip. di Fisica, Università di Roma "La Sapienza", 00185 Rome, Italy \\ ${ }^{5}$ INFN, sez. Roma, 00185 Rome, Italy \\ ${ }^{6}$ Laboratori Nazionali del Gran Sasso, INFN, Assergi, Italy \\ ${ }^{7}$ Key Laboratory of Particle Astrophysics, Institute of High Energy Physics, Chinese Academy of Sciences, P.O. Box 918/3, Beijing 100049, \\ China \\ ${ }^{8}$ University of Jing Gangshan, Ji'an, Jiangxi, China
}

Received: 24 February 2015 / Accepted: 20 May 2015 / Published online: 29 May 2015

(C) The Author(s) 2015. This article is published with open access at Springerlink.com

\begin{abstract}
In the present paper the results obtained in the investigation of possible diurnal effects for low-energy single-hit scintillation events of DAMA/LIBRA-phase1 (1.04 ton $\times$ year exposure) have been analysed in terms of an effect expected in case of dark matter (DM) candidates inducing nuclear recoils and having high cross-section with ordinary matter, which implies low DM local density in order to fulfill the DAMA/LIBRA DM annual modulation results. This effect is due to the different Earth depths crossed by those DM candidates during the sidereal day.
\end{abstract}

\section{Introduction}

The present DAMA/LIBRA experiment [1-13], as the former DAMA/NaI [14-43] has the main aim to investigate the presence of dark matter (DM) particles in the galactic halo by exploiting the model-independent DM annual modulation signature (originally suggested in Refs. [44,45]). In particular, they have cumulatively reached a model independent evidence at 9.3 $\sigma \mathrm{CL}$ for the presence of DM particles in the galactic halo by exploiting the DM annual modulation signature [4]. Recently the results obtained by investigating the presence of possible diurnal variation in the low-energy single-hit scintillation events collected by DAMA/LIBRAphase1 (1.04 ton $\times$ year exposure) have been released and analysed in terms of a DM second order model-independent

Deceased: S. d'Angelo.

a e-mail: rita.bernabei@roma2.infn.it effect due to the Earth diurnal rotation around its axis [12]. In particular, the data were analysed using the sidereal time referred to Greenwich, often called GMST. No diurnal variation with sidereal time has been observed at the reached level of sensitivity, which was not yet adequate to point out the effect searched for there. In the present paper those experimental data are analysed in terms of an effect - named "Earth Shadow Effect" - which could be expected for DM candidate particles inducing nuclear recoils; this effect would be induced by the variation - during the day - of the Earth thickness crossed by the DM particle in order to reach the experimental set-up. It is worth noting that a similar effect can be pointed out only for candidates with high cross-section with ordinary matter, which implies low DM local density in order to fulfill the DAMA/LIBRA DM annual modulation results. Such DM candidates could get trapped in substantial quantities in the Earth's core; in this case they could annihilate and produce secondary particles (e.g. neutrinos) and/or they could carry thermal energy away from the core, giving potentiality to further investigate them.

Preliminary investigations on DM candidates inducing diurnal variation were performed in Refs. [23,46,47] and more recently in Refs. [48-52].

\section{The Earth Shadow Effect}

During a sidereal day the Earth shields a terrestrial detector with a varying thickness, and this induces a variation of the flux of the DM candidates impinging the detector, mainly because of the modification of their velocity distribu- 


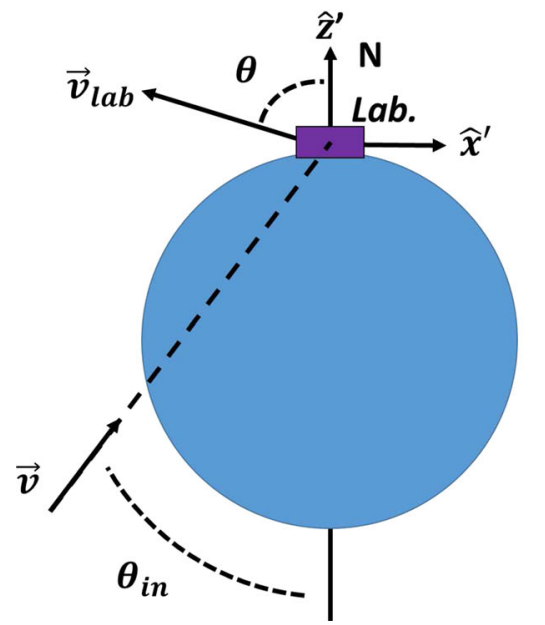

Fig. 1 Schematic view of the DM particles impinging direction on a detector; the $x^{\prime}, y^{\prime}, z^{\prime}$ represent the laboratory frame coordinate system. Left schematic representation of the correlation between the thickness, $d$, crossed by the considered DM candidates to reach a laboratory (hypotetically placed at the geographic North pole) and the DM

tion, $f(\vec{v})$. It is worth noting that this Earth Shadow Effect is very small and could be detectable only in case of candidates with high cross-section with ordinary matter (i.e. present in the galactic halo with small abundance).

The detector (and the hosting laboratory) velocity in the Galactic frame can be written as:

$\vec{v}_{l a b}(t)=\vec{v}_{L S R}+\vec{v}_{\odot}+\vec{v}_{r e v}(t)+\vec{v}_{r o t}(t)$,

where: (i) $\vec{v}_{L S R}$ is the velocity of the local standard of rest (LSR) because of the rotation of the Galaxy; (ii) $\vec{v}_{\odot}$ is the Sun peculiar velocity with respect to LSR; (iii) $\vec{v}_{\text {rev }}(t)$ is the velocity of the orbital motion of the Earth around the Sun and (iv) $\vec{v}_{\text {rot }}(t)$ is the velocity of the rotation of the Earth around its axis. The two latter terms change as function of the sidereal time, $t$. Using the galactic coordinate frame (that is $x$ axis towards the galactic center, $y$ axis following the rotation of the Galaxy and the $z$ axis towards the galactic North pole), one gets: $\vec{v}_{L S R}=\left(0, v_{0}, 0\right)$, where $v_{0}=(220 \pm 50) \mathrm{km} / \mathrm{s}$ (uncertainty at $90 \% \mathrm{CL}$ ) [22,53-55] is the local velocity, and $\vec{v}_{\odot}=(9,12,7) \mathrm{km} / \mathrm{s}[56]$.

The DM particles in the galactic halo have a velocity distribution $g(\vec{w})$, which depends on the considered galactic halo model. Reference [22] has shown many possible scenarios for the galactic halo; in the following we consider the isothermal halo model just because of its simplicity:

$g(\vec{w})=A e^{-\frac{w^{2}}{v_{0}^{2}}} \theta\left(v_{e s c}-|\vec{w}|\right)$,

with $A$ normalization constant and $v_{e s c}$ escape velocity, assumed in the following equal to $650 \mathrm{~km} / \mathrm{s}$, as often considered in literature; however, it is also affected by uncertainty. However, no sizeable differences are observed in the out-

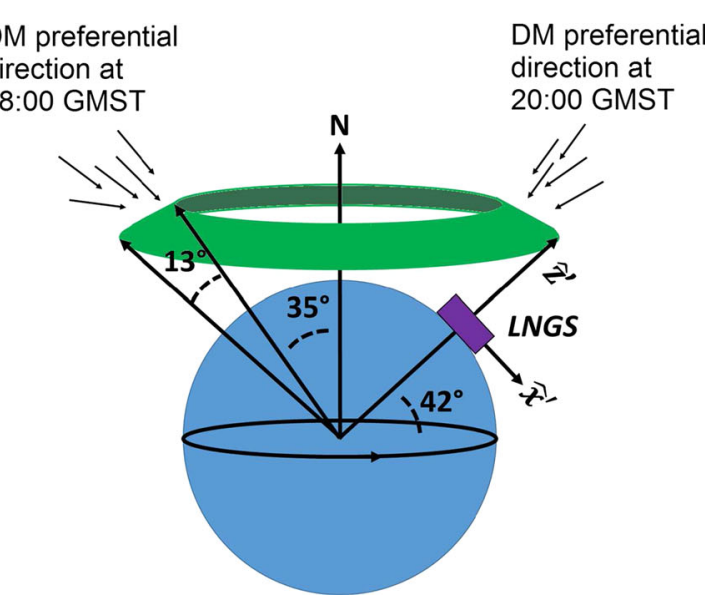

impinging angle, $\theta_{i n}$. Right schematic representation of the experimental condition considered in the text: detector placed at the Gran Sasso National Laboratory (LNGS) with the $z^{\prime}$ axis in the vertical direction and the $x^{\prime}$ axis pointing to the vernal equinox

come when a different value of $v_{e s c}=550 \mathrm{~km} / \mathrm{s}$ is considered, more closer to the $90 \%$ CL range of the RAVE Survey results [57]. In the laboratory frame the DM velocity distribution $f(\vec{v})$ is obtained from Eq. 2 straightforward since $\vec{w}=\vec{v}+\vec{v}_{l a b}$.

To evaluate the expected daily variation of the DM particles velocity distribution due to the Earth Shadow Effect, it is necessary to estimate the time dependence of the $\theta$ angle, the "zenith distance" of $\vec{v}_{l a b}$ (i.e. the distance between $\vec{v}_{l a b}$ and the zenith, see Fig. 1). This can be determined by astrophysical considerations studying the Earth's rotation around its axis.

The simplest way to calculate $\theta(t)$ is in the equatorial coordinate system [12] where the $\hat{e}_{1}^{\text {ecs }}$ axis is directed towards the vernal equinox, and $\hat{e}_{1}^{e c s}$ and $\hat{e}_{2}^{e c s}$ are on the equatorial plane; the $\hat{e}_{3}^{e c s}$ axis is towards the North pole. The righthanded convention is used. To work out the galactic coordinates of those versors, one considers: (i) the equatorial coordinates of the galactic North pole: $R A=192^{\circ} .859508$ and $D E=27^{\circ} .128336$, where $R A$ is the right ascension and $D E$ is the declination; (ii) the equatorial coordinates of the galactic center: $R A=266^{\circ} .405100$ and $D E=-28^{\circ} .936175$, evaluated at the Epoch J2000.0. In the galactic coordinates, those versors can be written as:

$\hat{e}_{1}^{e c s}=(-0.05487,0.49411,-0.86767)$

$\hat{e}_{2}^{e c s}=(-0.87344,-0.44483,-0.19808)$

$\hat{e}_{3}^{e c s}=(-0.48384,0.74698,0.45599)$.

We define $\vec{v}_{s}=\vec{v}_{L S R}+\vec{v}_{\odot}$. In this section, when a numerical calculation is employed, we assume $v_{0}=220 \mathrm{~km} / \mathrm{s}$; hence, $v_{s}=232.28 \mathrm{~km} / \mathrm{s}$, and in the equatorial coordinate system: $\theta_{e c s}=42^{\circ} .18$ (the "zenith distance") and $\varphi_{e c s}=-46^{\circ} .14$ 


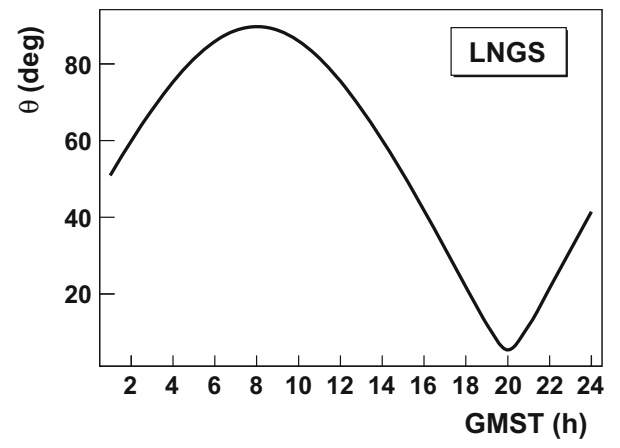

Fig. 2 The angle $\theta$ as a function of the sidereal time in the case of the LNGS (latitude $\lambda_{0}=13^{\circ} 34^{\prime} \mathrm{E}$, longitude $\phi_{0}=42^{\circ} 27^{\prime} \mathrm{N}$ ). The Earth shielding is maximum about at 8:00 $\mathrm{h}$ and minimum around 20:00 h; see text

(azimuth angle from $\hat{e}_{1}^{\text {ecs }}$ ). For simplicity in the following of this section in order to offer an estimate of the Earth Shadow Effect diurnal behaviour we consider $\vec{v}_{\text {rev }}(t)$ equal to its annual mean value, i.e. zero. To introduce the Earth motion around its axis, firstly we consider the simplest case of a laboratory at North pole and we define the horizontal coordinate system with $z^{\prime}$ axis directed as shown in Fig. 1, left and $x^{\prime}$ axis directed towards a given longitude $\lambda_{0}$. In this coordinate system ( $N$ label) the velocity of the Earth can be written as: $v_{l a b}^{N}=v_{s}, \theta_{N}=\theta_{e c s}$, and $\varphi_{N}=\varphi_{e c s}-\omega_{r o t}\left(t+t_{0}\right)=$ $-\left(\varphi_{0}+\omega_{\text {rot }}\left(t+t_{0}\right)\right)$, where $\varphi_{0}=-\varphi_{e c s}, \omega_{\text {rot }}=2 \pi / T_{d}$ with $T_{d}=1$ sidereal day, $t$ sidereal time referred to Greenwich, and $t_{0}=24 \lambda_{0} / 2 \pi$ sidereal hours.

The general case of $\vec{v}_{l a b}$ in a laboratory at latitude $\phi_{0}$ can be derived by rotating counterclockwise $\vec{v}_{l a b}^{N}$ around $\hat{y}^{\prime}$ of an angle $\alpha=\pi / 2-\phi_{0}$ :

$$
\begin{aligned}
\vec{v}_{\text {lab }}^{\lambda_{0}, \phi_{0}}= & R(\alpha) \vec{v}_{l a b}^{N} \\
= & \left(\begin{array}{ccc}
\cos \alpha & 0 & -\sin \alpha \\
0 & 1 & 0 \\
\sin \alpha & 0 & \cos \alpha
\end{array}\right) \\
& \times\left(\begin{array}{c}
v_{s} \sin \theta_{e c s} \cos \left(\varphi_{0}+\omega_{\text {rot }}\left(t+t_{0}\right)\right) \\
-v_{s} \sin \theta_{e c s} \sin \left(\varphi_{0}+\omega_{\text {rot }}\left(t+t_{0}\right)\right) \\
v_{s} \cos \theta_{e c s}
\end{array}\right) .
\end{aligned}
$$

Thus, $\theta(t)$ for a laboratory position, identified by the longitude $\lambda_{0}$ and latitude $\phi_{0}$, can be derived from $\cos \theta(t)=$ $\hat{v}_{l a b}^{\lambda_{0}, \phi_{0}} \cdot \hat{z}^{\prime}$ (see Fig. 1, right) obtaining:

$$
\begin{aligned}
\cos \theta(t)= & \sin \theta_{e c s} \cos \phi_{0} \cos \left(\omega_{\text {rot }}\left(t+t_{0}\right)+\varphi_{0}\right) \\
& +\cos \theta_{e c s} \sin \phi_{0} \\
= & \cos \psi \cos \phi_{0} \cos \left(\omega_{\text {rot }}\left(t+t_{0}\right)+\varphi_{0}\right) \\
& +\sin \psi \sin \phi_{0},
\end{aligned}
$$

where $\psi=\pi / 2-\theta_{e c s}=47^{\circ} .82$ is the altitude. In case of the LNGS, the longitude is $\lambda_{0}=13^{\circ} 34^{\prime} \mathrm{E}$ and the latitude is $\phi_{0}=42^{\circ} 27^{\prime} \mathrm{N}$, thus $t_{0}=0.904444$ sidereal hours. The $\theta(t)$ behaviour at LNGS is shown in Fig. 2. Note that, before detection, the DM particles at LNGS preferentially cross an Earth maximum thickness at about 08:00 h and an Earth minimum thickness at about 20:00 h (GMST in both cases).

\section{Deformation of the DM velocity distribution due to the Earth Shadow Effect}

To study the experimental data in terms of possible Earth Shadow Effect, a Monte Carlo code has been developed to simulate the propagation of the DM candidates elastically scattering off Earth nuclei in their travel in the Earth towards the underground experimental site. For such a purpose useful information has been gathered about the Earth composition and density. The Monte Carlo code numerically estimates the velocity distribution - in the laboratory coordinate system - of the impinging DM particles after having crossed the Earth; such velocity distribution depends on the mass of the DM candidate, on its cross-section on nucleons, on the initial unperturbed velocity distribution, on the sidereal time, and on the latitude and longitude of the laboratory: $f_{l a b}\left(v, t \mid m_{D M}, \sigma_{n}\right)$. Then, this velocity distribution has been used to evaluate - in an assumed framework - the expected counting rate as a function of the sidereal time in order to be compared with the experimental data.

In this section details are given about the assumptions adopted in the simulation, as in particular: the Earth model, the mean free path and path reconstruction of such DM candidate, the adopted interaction model (nuclear form factor, scaling law, etc.) and the $f_{l a b}\left(v, t \mid m_{D M}, \sigma_{n}\right)$ estimation.

\subsection{The Earth model}

An Earth model has been assumed in order to estimate the signal variation due to the Earth Shadow Effect; in particular, the matter density and composition of the Earth have to be considered. The simulation adopts a simplified Earth model starting by the preliminary reference Earth model (PREM) [58]. Just three main Earth layers with a constant density and homogeneous distribution in each one (these values are averaged over the PREM density distribution behaviour) are considered: (i) the Inner Core; (ii) the External Core; (iii) the Mantle. The densities and mass percentage for each layer are given in Table 1; in this simplified model the rare isotopes (mass percentage lower than $0.1 \%$ ) have been neglected.

\subsection{Interactions of the considered DM candidates and path reconstruction}

We assume that the considered DM candidates loss their energy elastically scattering off nuclei with spin-independent coupling. The mean free path in the $L$ th Earth's layer is: 
Table 1 Density values, $\rho_{L}$, and $i$ th nucleus mass percentage, $\delta_{i}$, adopted in the present calculations for the layers of the considered Earth model [58]

\begin{tabular}{llll}
\hline Layer $(L)$ & $\begin{array}{l}\mathrm{R}_{\text {min }}-\mathrm{R}_{\text {max }} \\
(\mathrm{km})\end{array}$ & $\begin{array}{l}\text { Mass percentage } \\
\left(\delta_{i}\right)\end{array}$ & $\begin{array}{l}\text { Density }\left(\rho_{L}\right) \\
\left(\mathrm{kg} / \mathrm{m}^{3}\right)\end{array}$ \\
\hline Inner Core & $0-1221.5$ & $\mathrm{Fe}(79 \%)$ & 12839 \\
External Core & $1221.5-3480$ & $\mathrm{Ni}(21 \%)$ & \\
& & $\mathrm{Fe}(86 \%)$ & 10901 \\
Mantle & $3480-6371$ & $\mathrm{Ni}(2 \%)$ & \\
& & $\mathrm{O}(44.9 \%)$ & 4605 \\
& & $\mathrm{Si}(21.6 \%)$ \\
& $\mathrm{Mg}(22.8 \%)$ & \\
& & $\mathrm{Fe}(5.8 \%)$ & \\
& & $\mathrm{Ca}(2.3 \%)$ \\
& & $\mathrm{Al}(2.2 \%)$ \\
& & $\mathrm{Na}(0.4 \%)$ & \\
& &
\end{tabular}

$\lambda_{L}=\frac{1}{\sum_{i=1}^{N} \sigma_{D M, i} n_{i}}$,

where: (i) $n_{i}=\frac{\rho_{L}}{m_{i}} \delta_{i}$ is the number density of the $i$ th nuclei in the Earth's layer composed by $N$ nuclear species; (ii) $\sigma_{D M, i}$ is the cross-section on the $i$ th nucleus and $m_{i}$ is the mass of the $i$ th nucleus; (iii) $\rho_{L}$ is the layer's density; (iv) $\delta_{i}$ is the $i$ th nucleus mass percentage in the layer. We considered a coherent scattering and a scaling law ${ }^{1}$ :

$\sigma_{D M, i}=\sigma_{n} A_{i}^{2} \frac{\mu_{i}^{2}}{\mu_{n}^{2}}$

where: (i) $A_{i}$ is the mass number of nucleus $i$; (ii) $\sigma_{n}$ is the DM candidate-nucleon cross-section; (iii) $\mu_{i}\left(\mu_{n}\right)$ is the DM candidate - nucleus (nucleon) reduced mass. Thus, assuming $m_{i} \simeq A_{i} m_{n}$, one can write:

$$
\frac{1}{\lambda_{L}}=\frac{\sigma_{n} \rho_{L}}{m_{n}} \sum_{i=1}^{N} A_{i}^{3} \delta_{i}\left(\frac{m_{D M}+m_{n}}{m_{D M}+A_{i} m_{n}}\right)^{2} .
$$

For simplicity, the deflection of the DM particles crossing the Earth is neglected (i.e. a linear trajectory is considered); in this assumption the Earth thickness crossed by those DM candidates, $d$, depends only on the impinging angle with respect to the detector, $\theta_{i n}$, according to the relation:

$d=2 R_{\oplus} \cos \left(\theta_{i n}\right)$,

1 This equation holds in the limit where the form factor can be neglected. For high velocity, high mass DM candidate crossing the Inner Core it is only an approximation. For simplicity we do not consider further this issue here, while the form factor is considered in obtaining the energy loss (see later). with $R_{\oplus}$ Earth radius. This $d$ value is the sum of the distances passed through by the DM candidate in each layer: $d=$ $\sum_{L} d_{L}$. Defining the maximum radii of the Earth layers $R_{i c}$, $R_{e c}$ and $R_{m}=R_{\oplus}$ for Inner Core, External Core and Mantle respectively (see Table 1), the number of layers crossed by DM particles in the considered schema is: 0 for $\theta_{i n} \geq 90^{\circ}$; 1 for $33^{\circ} .11 \leq \theta_{i n}<90^{\circ}$; 3 for $11^{\circ} .05 \leq \theta_{i n}<33^{\circ} .11 ; 5$ for $0^{\circ} \leq \theta_{i n}<11^{\circ} .05$ (considering that $\arcsin \left(R_{e c} / R_{\oplus}\right)=$ $33^{\circ} .11$ and $\left.\arcsin \left(R_{i c} / R_{\oplus}\right)=11^{\circ} .05\right)$.

Thus, in this scenario, the DM particles move in each Earth's layer with a mean free path $\lambda_{L}$, that mainly depends on the interaction cross section $\sigma_{n}$ (see Eq. 8). The number of interactions in each layer, $n_{h i t}$, has been estimated as:

- Case 1, high interaction cross sections, $d_{L} \geq 50 \lambda_{L}: n_{\text {hit }}$ is relatively high and follows a gaussian distribution with mean value and variance equal to $d_{L} / \lambda_{L}$;

- Case 2, small interaction cross sections, $d_{L}<50 \lambda_{L}$ : a step-by-step approach has been adopted in the simulation. The path between two consecutive interactions, $x_{k}$, follows the distribution $\lambda_{L}^{-1} e^{-\left(x_{k} / \lambda_{L}\right)}$; it can be used to propagate the particle within the layer as long as $\sum_{k}^{n h i t} x_{k} \leq d_{L}$.

In the considered scenario the DM candidate particles interact via SI elastic scattering on nuclei; thus, their energy-loss for each interaction is given by the induced nuclear recoil energy:

$E_{R}=E_{i n} r\left(\frac{1-\cos \theta^{*}}{2}\right)$,

where $E_{i n}=m_{D M} v^{2} / 2$ and $v$ are the DM energy and the velocity before the interaction, $\theta^{*}$ is the angle of diffusion in the center of mass and $r$ is a kinematic factor:

$r=\frac{4 m_{D M} m_{i}}{\left(m_{D M}+m_{i}\right)^{2}}$.

The interaction cross-section is given by [18]:

$\frac{d \sigma_{D M, i}}{d E_{R}}\left(v, E_{R}\right)=\frac{d \sigma_{D M, i}}{d E_{R}}(v, 0) F_{i}^{2}\left(E_{R}\right)$,

with $F_{i}^{2}\left(E_{R}\right)$ nuclear form factor. In addition, for a given velocity $v$ :

$\frac{d \sigma_{D M, i}}{d E_{R}}(v, 0)=\frac{d \sigma_{D M, i}}{d \Omega} \times \frac{d \Omega}{d E_{R}}$,

with (also see Eq. 10):

$\frac{d \sigma_{D M, i}}{d \Omega}=\frac{\sigma_{D M, i}}{4 \pi}, \quad \frac{d E_{R}}{d \Omega}=\frac{r E_{i n}}{4 \pi}$.

Hence, assuming the cross section scaling law given in Eq. 7, the interaction cross-section can be written as: 

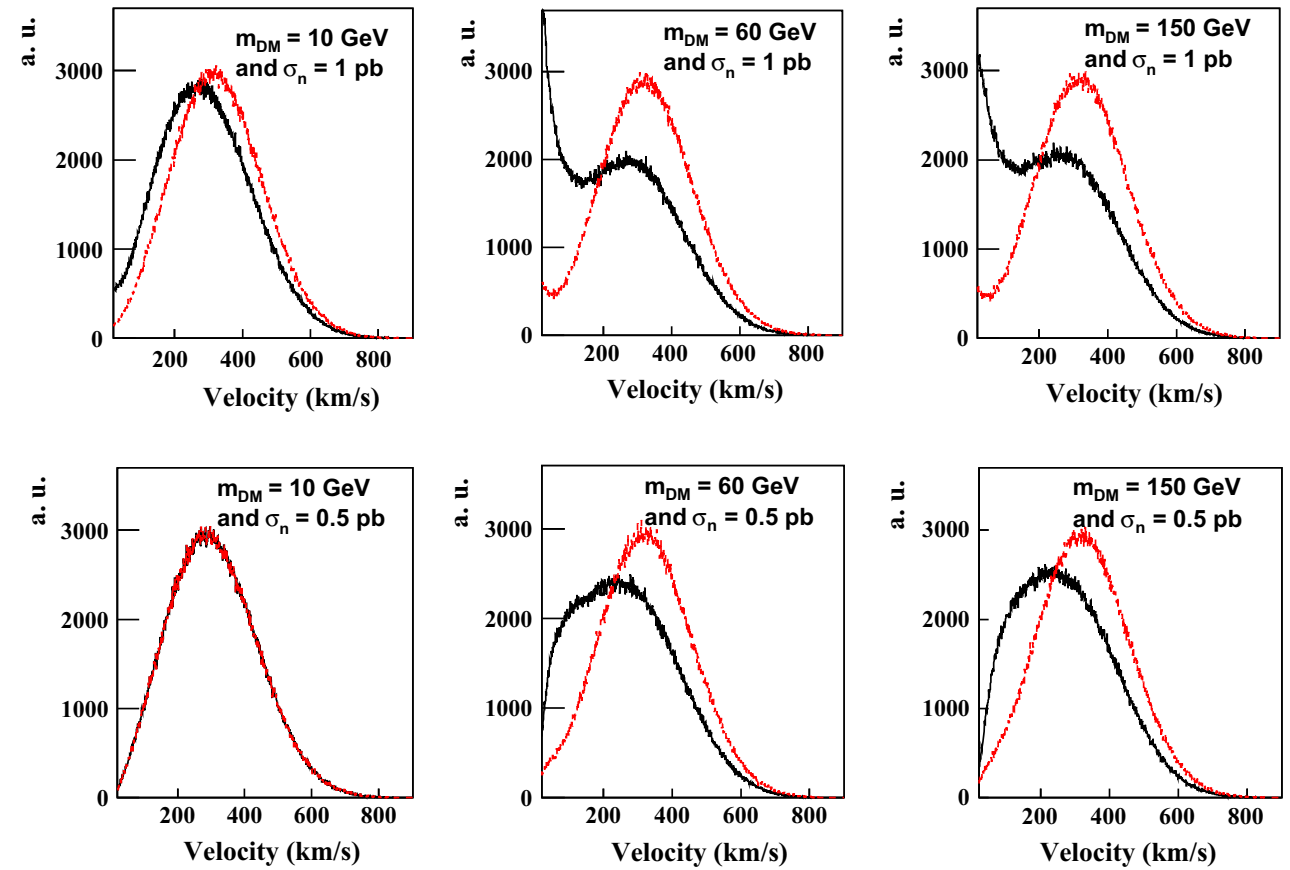

Fig. 3 Some examples of simulated $f_{l a b}\left(v, t \mid m_{D M}, \sigma_{n}\right)$ in arbitrary units for some $m_{D M}$ and $\sigma_{n}$ values when taking into account the Earth Shadow Effect. In these plots: (i) the considered Galactic Halo model is an isothermal sphere with $v_{0}=220 \mathrm{~km} / \mathrm{s}$; (ii) the velocity distribu-

$$
\frac{d \sigma_{D M, i}}{d E_{R}}\left(v, E_{R}\right)=\frac{\sigma_{D M, i}}{r E_{i n}} F_{i}^{2}\left(E_{R}\right)=\sigma_{n} A_{i}^{2} \frac{m_{i}}{2 \mu_{n}^{2} v^{2}} F_{i}^{2}\left(E_{R}\right)
$$

Thus, after one interaction, such a DM particle loses an energy in the range $0 \leq E_{R} \leq r E_{i n}$ with distribution given in Eq. 15. In the following we assume for the nuclear form factor the Helm formula ${ }^{2}[59,60]$ :

$F_{i}\left(q r_{0}\right)=\frac{3\left[\sin \left(q r_{0}\right)+q r_{0} \cos \left(q r_{0}\right)\right]}{\left(q r_{0}\right)^{3}} e^{-\frac{1}{2} q^{2} s^{2}}$,

where $r_{0}=\sqrt{r_{i}^{2}-5 s^{2}}, r_{i}=1.2 A_{i}^{1 / 3} \mathrm{fm}, s \simeq 1 \mathrm{fm}$ and $q^{2}=2 m_{i} E_{R}$.

In the simulation, once $n_{\text {hit }}$ has been evaluated, the energy loss of the considered DM particle, $E_{R}$, is estimated for each interaction and the output velocity is:

$v_{f}=\sqrt{v^{2}-\frac{2 E_{R}}{m_{D M}}}$

\footnotetext{
$\overline{2}$ It is important to remark that the spin independent form factor depends on the target nucleus and there is not an universal formulation for it. Many profiles are available in literature and whatever profile needs some parameters whose value are also affected by some uncertainties. The form factor profiles can differ - in some intervals of the transferred momentum - by orders of magnitude and the chosen profile strongly affects whatever model dependent results [25].
}

tion at GMST hour 8:00 (continuous black line) and 20:00 (dashed-red on-line-line - corresponding to the maximum and the minimum of the Earth Shadow Effect in case of a target-detector placed at LNGS

Therefore, the net effect is a modification of the velocity distribution, $f_{l a b}\left(v, t \mid m_{D M}, \sigma_{n}\right)$; Fig. 3 shows some examples of the obtained velocity distribution for a detector located at LNGS.

\section{The expected interaction rate}

In the SI coupling scenario, considered here, the DM candidates scatter off the nuclei in the detector. Their expected interaction rate as a function of the nuclear recoil energy, $E_{R}$, for a mono-atomic ( $i$ nucleus) detector is:

$$
\begin{aligned}
\frac{d N_{i}}{d E_{R}}= & N_{T} \int_{v_{\min }\left(E_{R}\right)}^{\infty} \frac{\rho v}{m_{D M}} f_{l a b}(v) \\
& \times \frac{d \sigma_{D M, i}}{d E_{R}}(v, 0) F_{i}^{2}\left(E_{R}\right) d v,
\end{aligned}
$$

where $N_{T}$ is the number density of the target nuclei in the detector and $\rho=\xi \rho_{0}$ is the DM particles density in the galactic halo ( $\xi$ is the relative abundance and $\rho_{0}$ the overall DM density in the galactic halo). The integral is calculated over all the possible DM particle velocities in the laboratory frame considering the distribution $f_{l a b}(v)=f_{l a b}\left(v, t \mid m_{D M}, \sigma_{n}\right)$. The minimal velocity providing $E_{R}$ recoil energy is $v_{\min }\left(E_{R}\right)=\sqrt{\frac{E_{R} m_{i}}{2 \mu_{i}^{2}}}$. The galactic escape velocity is included in the $f(v)$ definition. Using Eq. 15 , the expected rate can be rewritten as: 


$$
\begin{aligned}
\frac{d N_{i}}{d E_{R}}= & \xi \sigma_{n} \frac{N_{T} \rho_{0}}{m_{D M}} A_{i}^{2} \frac{m_{i}}{2 \mu_{n}^{2}} F_{i}^{2}\left(E_{R}\right) \\
& \times \int_{v_{\min }\left(E_{R}\right)}^{\infty} \frac{f_{l a b}\left(v, t \mid m_{D M}, \sigma_{n}\right)}{v} d v \\
= & \xi \sigma_{n} \frac{d N_{i}^{\prime}}{d E_{R}}\left(E_{R}, t \mid m_{D M}, \sigma_{n}\right) .
\end{aligned}
$$

Generalizing to detectors with more than one kind of target nuclei [as e.g. in the case of the $\mathrm{NaI}(\mathrm{Tl})$ considered here], the expected experimental rate is:

$$
\frac{d R}{d E_{d e t}}=\xi \sigma_{n} \frac{d R^{\prime}}{d E_{d e t}}\left(E_{d e t}, t \mid m_{D M}, \sigma_{n}\right),
$$

where:

$$
\begin{aligned}
& \frac{d R^{\prime}}{d E_{d e t}}\left(E_{d e t}, t \mid m_{D M}, \sigma_{n}\right) \\
& =\int G\left(E_{d e t}, E^{\prime}\right)\left[\sum_{i} \int K_{i}\left(E^{\prime} \mid E_{R}\right)\right. \\
& \left.\quad \times \frac{d N_{i}^{\prime}}{d E_{R}}\left(E_{R}, t \mid m_{D M}, \sigma_{n}\right) d E_{R}\right] d E^{\prime} ;
\end{aligned}
$$

the $G\left(E_{\text {det }}, E^{\prime}\right)$ kernel takes into account the detector's energy resolution (generally through a gaussian convolution) and the $K_{i}\left(E^{\prime} \mid E_{R}\right)$ kernel takes into account the energy transformation of the nuclear recoil energy in $\mathrm{keV}$ electron equivalent. For example the latter kernel can be written in the simplest case of a constant quenching factor, $q_{i}$, as: $K_{i}\left(E^{\prime} \mid E_{R}\right)=\delta\left(E^{\prime}-q_{i} E_{R}\right)$. For a discussion about the quenching factors see Refs. [5,8].

The expected differential rate - as well as $f_{l a b}\left(v, t \mid m_{D M}\right.$, $\sigma_{n}$ ) - depends on the time through three different effects: (i) the time dependence of the Earth's orbital motion velocity, $\vec{v}_{r e v}(t)$ (see Eq. 1); (ii) the time dependence of the Earth's rotation velocity around its axis, $\vec{v}_{\text {rot }}(t)$ (see Eq. 1); (iii) the possible Earth Shadow Effect which depends on $\sigma_{n}$. Details about the first two effects, responsible of the modelindependent annual and diurnal modulation of the DM signal rate, respectively, are reported in Ref. [12]. Following the same approach the expected rate in an energy interval $\Delta E_{k}$ can be written as ${ }^{3}$ :

$$
\begin{aligned}
S_{k}(t)= & \int_{\Delta E_{k}} \xi \sigma_{n}\left(\frac{d R^{\prime}}{d E_{d e t}}\right)\left(E_{d e t}, t \mid m_{D M}, \sigma_{n}\right) d E_{d e t} \\
= & \xi \sigma_{n}\left[S_{0, k}^{\prime}\left(m_{D M}, \sigma_{n}\right)\right. \\
& +S_{m, k}^{\prime}\left(m_{D M}, \sigma_{n}\right) \cos \left(\omega\left(t-t_{0}\right)\right) \\
& +S_{d, k}^{\prime}\left(m_{D M}, \sigma_{n}\right) \cos \left(\omega_{r o t}\left(t-t_{d}\right)\right) \\
& \left.+S_{d, s h, k}^{\prime}\left(m_{D M}, \sigma_{n}, t\right)\right]
\end{aligned}
$$

\footnotetext{
3 Here only the first order terms are shown (i.e. the interference terms are omitted).
}

where: (i) $S_{0, k}^{\prime}\left(m_{D M}, \sigma_{n}\right)$ is the time independent component of the expected signal; (ii) $S_{m, k}^{\prime}\left(m_{D M}, \sigma_{n}\right)$ is the annual modulation amplitude, $\omega=2 \pi / T$ with $T=1 \mathrm{yr}, t_{0} \simeq$ June 2nd; (iii) $S_{d, k}^{\prime}\left(m_{D M}, \sigma_{n}\right)$ is the diurnal modulation amplitude, $\omega_{\text {rot }}=2 \pi / T_{d}$ with $T_{d}=1$ sidereal day, $t_{d}$ for the case of a detector at the Gran Sasso longitude ranges from 13.94 to $14.07 \mathrm{~h}$ depending on the $v_{0}$ value (see Ref. [12]); (iv) $S_{d, s h, k}^{\prime}\left(m_{D M}, \sigma_{n}, t\right)$ (whose average value over $T_{d}$ is null) takes into account the signal variation as a function of the sidereal time due to a possible Earth Shadow Effect.

The ratio $R_{d y}=S_{d, k}^{\prime}\left(m_{D M}, \sigma_{n}\right) / S_{m, k}^{\prime}\left(m_{D M}, \sigma_{n}\right)$ is model independent and it is $R_{d y} \simeq 0.016$ at LNGS latitude; thus, considering the DAMA/LIBRA-phase1 experimental result on the DM annual modulation, the expected $\xi \sigma_{n} S_{d, k}^{\prime}\left(m_{D M}, \sigma_{n}\right)$ is order of $10^{-4}$ counts per sidereal day per $\mathrm{kg}$ per $\mathrm{keV}\left(\mathrm{cpd}_{\text {sid }} / \mathrm{kg} / \mathrm{keV}\right.$, hereafter) [12]. The reached experimental sensitivity of DAMA/LIBRA-phase1 [12] is not yet enough to observe such a diurnal modulation amplitude; in fact, in the (2-4) keV energy interval considered here, the experimental diurnal modulation amplitude from DAMA/LIBRA-phase 1 data is $(2.0 \pm 2.1) \times$ $10^{-3} \mathrm{cpd}_{\text {sid }} / \mathrm{kg} / \mathrm{keV}$ [12] $\left(<5.5 \times 10^{-3} \mathrm{cpd}_{\text {sid }} / \mathrm{kg} / \mathrm{keV}\right.$, $90 \% \mathrm{CL})$. Thus, in the following we do not further approach it.

Few examples of the $S_{d, s h, k}^{\prime}(t)$ behavior for $m_{D M}=30$ $\mathrm{GeV}$ and for different values of the cross-section in the given framework are shown in Fig. 4. For clarity, in the explicative case of $\sigma_{n}=10 \mathrm{pb}$ and $\xi=1.1 \times 10^{-8}$ so that $\xi \sigma_{n}=1.1 \times 10^{-7} \mathrm{pb}$ is compatible with the DAMA/LIBRAphase1 DM annual modulation result, the obtained amplitude of $\xi \sigma_{n} S_{d, s h, k}^{\prime}(t)$ is of order of $3 \times 10^{-2} \mathrm{cpd}_{s i d} / \mathrm{kg} / \mathrm{keV}$. Such value can be studied in DAMA/LIBRA-phase1 (see later).

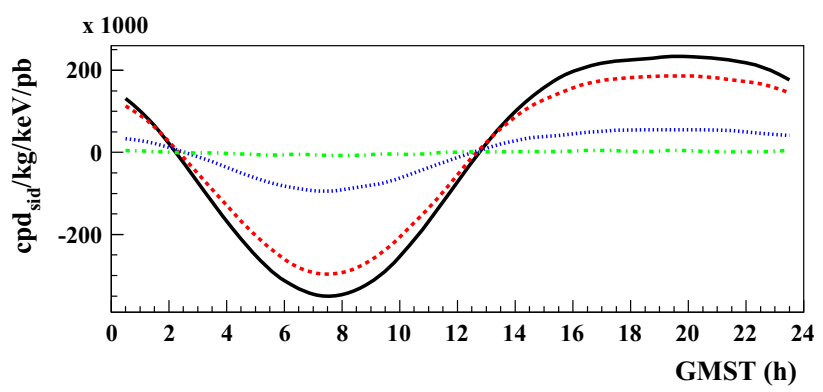

Fig. 4 Examples of expected $S_{d, s h, k}^{\prime}\left(m_{D M}, \sigma_{n}, t\right)$ obtained following the approach described in the text. The $\mathrm{Q}_{I}$ quenching factor and the set A of the parameters' values in presence of the channeling effect have been considered (see text); moreover, $v_{0}=220 \mathrm{~km} / \mathrm{s}$. The energy interval, considered here, is (2-4) $\mathrm{keV}$ and the $m_{D M}=30 \mathrm{GeV}$. The obtained $S_{d, s h, k}^{\prime}\left(m_{D M}, \sigma_{n}, t\right)$ are shown for the cases: (i) $\sigma_{n}=10 \mathrm{pb}$ (continuous black line); (ii) $\sigma_{n}=1 \mathrm{pb}$ (dashed-red on-line-line); (iii) $\sigma_{n}=0.1 \mathrm{pb}$ (dotted-blue on-line-line $) ;$ iv) $\sigma_{n}=0.01 \mathrm{pb}$ (dot-dashedgreen on-line-line) 


\section{Data analysis}

The results, obtained by analysing in the framework of the Earth Shadow Effect the DAMA/LIBRA-phase1 (total exposure 1.04 ton $\times$ year) data, essentially depend on the most sensitive (2-4) keV interval; thus, this is the energy region considered here.

In the present analysis, as in Refs. [5,32], three possibilities for the $\mathrm{Na}$ and I quenching factors have been considered: $\left(\mathrm{Q}_{I}\right)$ the quenching factors of $\mathrm{Na}$ and I "constants" with respect to the recoil energy $E_{R}: q_{N a} \simeq 0.3$ and $q_{I} \simeq 0.09$ as measured by DAMA with neutron source integrated over the $6.5-97$ and the $22-330 \mathrm{keV}$ recoil energy range, respectively [15]; $\left(\mathrm{Q}_{I I}\right)$ the quenching factors evaluated as in Ref. [61] varying as a function of $E_{R} ;\left(\mathrm{Q}_{I I I}\right)$ the quenching factors with the same behaviour of Ref. [61], but normalized in order to have their mean values consistent with $\mathrm{Q}_{I}$ in the energy range considered there.

Another important effect is the channeling of low energy ions along axes and planes of the $\mathrm{NaI}(\mathrm{Tl})$ DAMA crystals. This effect can lead to an important deviation, in addition to the other uncertainties discussed above. In fact, the channeling effect in crystals implies that a fraction of nuclear recoils are channeled and experience much larger quenching factors than those derived from neutron calibration (see $[5,30]$ for a discussion of these aspects). Since the channeling effect cannot be generally pointed out with neutron measurements as already discussed in details in Ref. [30], only modeling has been produced up to now. In particular, the modeling of the channeling effect described by DAMA in Ref. [30] is able to reproduce the recoil spectrum measured at neutron beam by some other groups (see Ref. [30] for details). For completeness, we mention an alternative channeling model, as that of Ref. [62], where larger probabilities of the planar channeling are expected. Moreover, we mention the analytic calculation claiming that the channeling effect holds for recoils coming from outside a crystal and not from recoils produced inside it, due to the blocking effect [63]. Nevertheless, although some amount of blocking effect could be present, the precise description of the crystal lattice with dopant and trace contaminants is quite difficult and analytical calculations require some simplifications which can affect the result. Because of the difficulties of experimental measurements and of theoretical estimate of this channeling effect, in the following it will be either included or not in order to give idea on the related uncertainty.

Thus, the data analysis has been repeated in some discrete cases which allow us to account for the uncertainties on the quenching factors and on the parameters used in the nuclear form factors. The first case (set A) is obtained considering the mean values of the parameters of the used nuclear form factors (see above and Ref. [25]) and of the

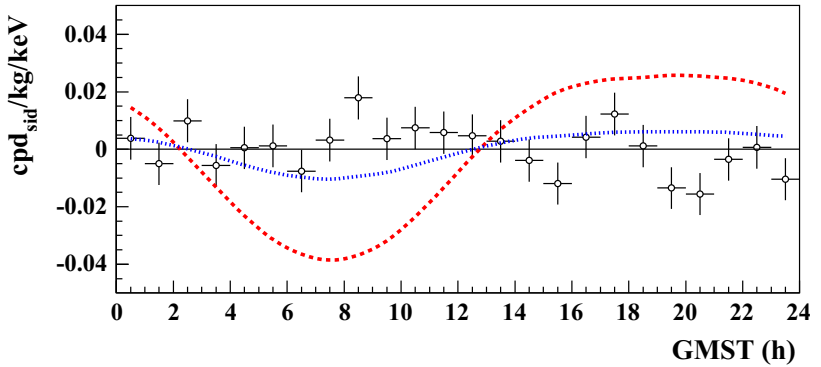

Fig. 5 Experimental model-independent diurnal residual rate of the single-hit scintillation events measured by DAMA/LIBRA-phase 1 in the (2-4) keV energy interval (crosses) as function of the sidereal time [12], superimposed to two examples of expectations obtained by MC simulation. The template curves are obtained in the same scenario as in Fig. 4, considering $\xi \sigma_{n}=1.1 \times 10^{-7} \mathrm{pb}$ (compatible with the DAMA/LIBRA-phase1 DM annual modulation result): (i) the dashed (red on-line) line is obtained for $\sigma_{n}=10 \mathrm{pb}$ (and, thus, $\xi=1.1 \times 10^{-8}$ ); (ii) the dotted (blue on-line) line is obtained for $\sigma_{n}=0.1 \mathrm{pb}$ (and, thus, $\xi=1.1 \times 10^{-6}$ ). The latter is compatible with the absence of diurnal rate variation in DAMA/LIBRA-phase1, while the former is not

quenching factors. The set B adopts the same procedure as in Refs. [20,21], by varying (i) the mean values of the measured ${ }^{23} \mathrm{Na}$ and ${ }^{127} \mathrm{I}$ quenching factors up to +2 times the errors; (ii) the nuclear radius, $r_{i}$, and the nuclear surface thickness parameter, $s$, in the form factor from their central values down to $-20 \%$. In the last case (set C) the Iodine nucleus parameters are fixed at the values of case B, while for the Sodium nucleus one considers: (i) ${ }^{23} \mathrm{Na}$ quenching factor at the lowest value measured in literature; (ii) the nuclear radius, $r_{i}$, and the nuclear surface thickness parameter, $s$, in the SI form factor from their central values up to $+20 \%$. Finally, three values of $v_{0}$ have been considered: (i) the mean value: $220 \mathrm{~km} / \mathrm{s}$, and (ii) two extreme cases: 170 and $270 \mathrm{~km} / \mathrm{s}$.

Because of the large number of the needed simulations, the mass of the DM candidate and of the cross section on nucleon have been discretized as in the following: six $m_{D M}$ $(5,10,30,60,100$ and $150 \mathrm{GeV})$ and eight $\sigma_{n}(10,5,1,0.5$, $0.1,0.05,0.01$ and $0.005 \mathrm{pb}$ ).

The expectations are compared with the experimental model-independent diurnal residual rate of the single-hit scintillation events, measured by DAMA/LIBRA-phase1 in the (2-4) $\mathrm{keV}$ energy interval, as function of the sidereal time (see in Fig. 2 of Ref. [12]). Two examples of expected signals are reported in Fig. 5. Here, the used sidereal time bin is $1 \mathrm{~h}$ (24 time bins in the sidereal day) and the experimental residuals are: $S_{d}^{\text {exp }}\left(t_{i}\right) \pm \sigma_{d, i}$. We compute the $\chi^{2}$ quantity:

$$
\begin{aligned}
\chi^{2} & =\sum_{i=1}^{24} \frac{\left(S_{d}^{\exp }\left(t_{i}\right)-\xi \sigma_{n} S_{d, s h, k}^{\prime}\left(m_{D M}, \sigma_{n}, t_{i}\right)\right)^{2}}{\sigma_{d, i}^{2}}= \\
& =\chi_{0}^{2}-2 B \xi+A \xi^{2},
\end{aligned}
$$


where

$$
\begin{aligned}
& \chi_{0}^{2}=\sum_{i=1}^{24} \frac{\left(S_{d}^{\text {exp }}\left(t_{i}\right)\right)^{2}}{\sigma_{d, i}^{2}} \\
& B\left(m_{D M}, \sigma_{n}\right)=\sigma_{n} \sum_{i=1}^{24} \frac{S_{d}^{e x p}\left(t_{i}\right) \times S_{d, s h, k}^{\prime}\left(m_{D M}, \sigma_{n}, t_{i}\right)}{\sigma_{d, i}^{2}} \\
& A\left(m_{D M}, \sigma_{n}\right)=\sigma_{n}^{2} \sum_{i=1}^{24} \frac{\left(S_{d, s h, k}^{\prime}\left(m_{D M}, \sigma_{n}, t_{i}\right)\right)^{2}}{\sigma_{d, i}^{2}} .
\end{aligned}
$$

The $S_{d, s h, k}^{\prime}\left(m_{D M}, \sigma_{n}, t_{i}\right)$ have been evaluated for each set of parameters described above.

The $\chi^{2}$ of Eq. 23 is function of only one parameter, $\xi$. Since the data do not show the presence of significant diurnal variation in the counting rate as already described in Ref. [12], only upper limits for $\xi$ are allowed, once given $m_{D M}$ and $\sigma_{n}$. We can define:

$\Delta \chi^{2}\{\xi\}=\chi^{2}\{\xi\}-\chi_{0}^{2}$.

The $\Delta \chi^{2}$ is a $\chi^{2}$ with one degree of freedom and is used to determine the upper limit of $\xi$ parameter at $2 \sigma \mathrm{CL}$.

Two examples to describe the followed procedure are reported in Fig. 6, where the excluded regions (above the dotted lines) in the $\xi$ vs $\sigma_{n}$ plane for the cases of $m_{D M}=10$ and $60 \mathrm{GeV}$ are shown as obtained on the basis of the Earth Shadow Effect in the given model framework.

The upper limits on $\xi$ can be compared with the positive results from the DM annual modulation signature achieved
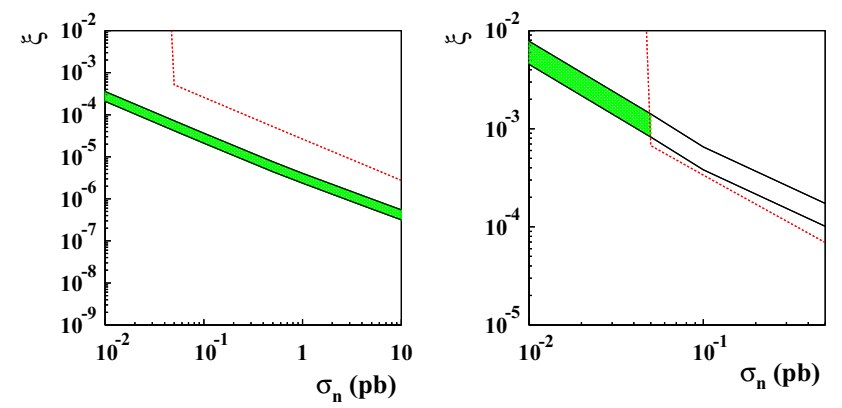

Fig. 6 Examples of comparisons at $2 \sigma$ CL between allowed regions from the DM annual modulation results of DAMA/LIBRA-phase1 (black continuous lines) and exclusion limits from the Earth Shadow Effect (dotted-red on-line-line). In both cases the quenching factors $\mathrm{Q}_{I}$, including the channeling effect, and the set $\mathrm{B}$ of the parameters have been considered (see text) for $v_{0}=220 \mathrm{~km} / \mathrm{s}$ and for two DM masses. Left $m_{D M}=10 \mathrm{GeV}$, the upper limits on $\xi$ do not constrain the results of annual modulation. Right $m_{D M}=60 \mathrm{GeV}$, the upper limits on $\xi$ do exclude the band with $\sigma_{n}>0.05 \mathrm{pb}$ and $\xi>10^{-3}$ for the considered model framework. The combined allowed regions are reported as shaded-green on-line-area by DAMA. ${ }^{4}$ In particular, DAMA/LIBRA-phase1 reports an annual modulation amplitude in the (2-4) keV energy interval: $S_{m}^{\text {exp }}=(0.0167 \pm 0.0022) \mathrm{cpd} / \mathrm{kg} / \mathrm{keV}$, corresponding to $7.6 \sigma \mathrm{CL}$ [4].

Here for each set of parameters described above, one can evaluate (see e.g. Eq. 22) the $\xi \sigma_{n}$ allowed values as:

$\xi \sigma_{n}=\frac{S_{m}^{\exp }}{S_{m,(2-4) k e V}^{\prime}\left(m_{D M}, \sigma_{n}\right)}$.

This corresponds, once including the experimental uncertainties on $S_{m}^{\exp }$, to a band in the $\xi$ vs $\sigma_{n}$ plane (within the continuous solid line). In Fig. 6 such bands at $2 \sigma$ CL are reported. One can see that for the scenario considered there and for $m_{D M}=10 \mathrm{GeV}$ the upper limits on $\xi$ do not constrain the results of the DM annual modulation. On the contrary, for $m_{D M}=60 \mathrm{GeV}$ the upper limits on $\xi$ do exclude the band with $\sigma_{n}>0.05 \mathrm{pb}$ and $\xi>10^{-3}$. The shaded bands in Fig. 6 corresponds to the allowed regions in the $\xi$ vs $\sigma_{n}$ plane, for the given $m_{D M}$, from the combined analyses of the DM annual modulation result and of the Earth Shadow Effect in the considered framework.

Finally, for each considered set of parameters the threedimensional allowed region - calculated as described abovein the parameter's space: $\xi, \sigma_{n}, m_{D M}$, is depicted as a surface in Figs. 7, 8 and 9, for $v_{0}$ equal to $170,220,270 \mathrm{~km} / \mathrm{s}$, respectively. We note that the "thickness" of the allowed regions around the shown surfaces is $\leq \pm 30 \%$; therefore, for simplicity it is not represented in these figures.

Finally, we recall that other uncertainties not considered in the present paper are present. For example, including other possible halo models sizeable differences are expected in the results as shown e.g. in Refs. [5,22].

\section{Conclusions}

The Earth Shadow Effect has been investigated in a given framework considering the model independent results on possible diurnal variation of the low-energy rate of the singlehit scintillation events in the DAMA/LIBRA-phase1 data (exposure: 1.04 ton $\times$ year) reported in Ref. [12]. For the considered DM candidates having high interaction crosssections and very small halo fraction the obtained results constrain at $2 \sigma \mathrm{CL}$, in the considered scenario, the $\xi, \sigma_{n}$ and $m_{D M}$ parameters (see Figs. $7,8,9$ ) when including the positive results from the DM annual modulation analysis of the DAMA/LIBRA-phase1 data [4]. For example, in the con-

\footnotetext{
${ }^{4}$ We recall that DAMA/LIBRA and the former DAMA/NaI have cumulatively reached a model independent evidence at $9.3 \sigma$ CL for the presence of DM particles in the galactic halo on the basis of the exploited DM annual modulation signature [4].
} 
(a)

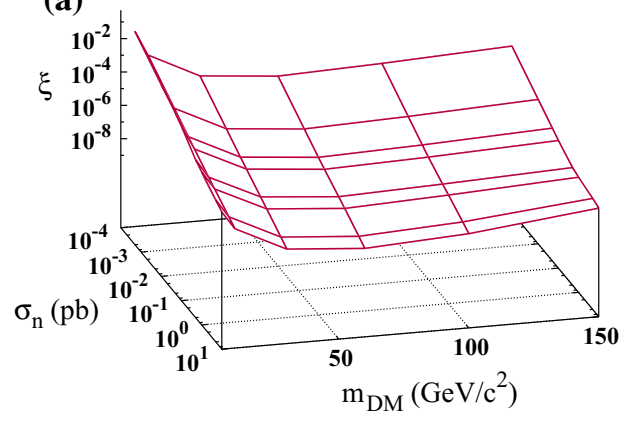

(c)

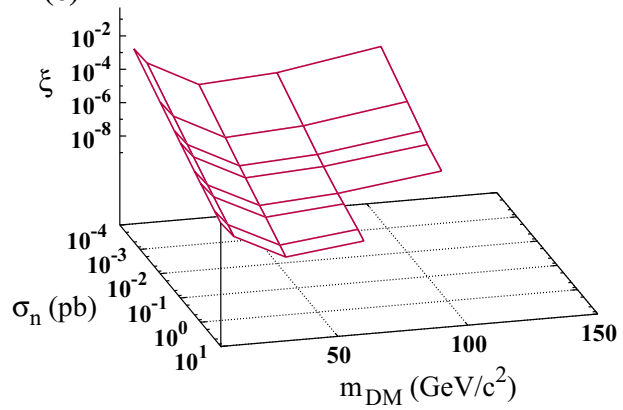

Fig. 7 Examples of the mean values of the allowed region of $\xi$ as function of $\sigma_{n}$ and $m_{D M}$, represented as an allowed surface (see text). The plots have been obtained for $v_{0}=170 \mathrm{~km} / \mathrm{s}$ in the following scenarios: a the quenching factors $\mathrm{Q}_{I}$, without channeling and marginalizing over the parameters sets A, B, C; $\mathbf{b}$ as in case $\mathbf{a}$ including the channeling

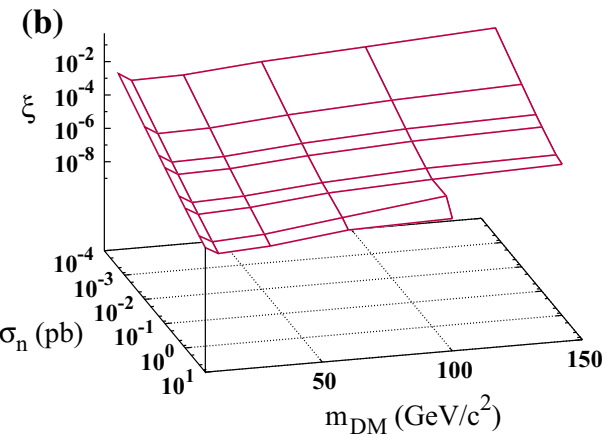

(d)

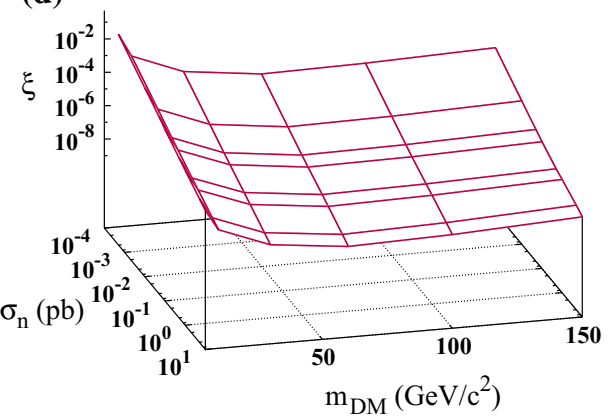

effect; $\mathbf{c}$ the quenching factors $\mathrm{Q}_{I I} ; \mathbf{d}$ the quenching factors $\mathrm{Q}_{I I I}$. We note that the "thickness" of the allowed regions around the surfaces is $\leq \pm 30 \%$; therefore, for simplicity it is not represented in these figures. Finally, we recall that other uncertainties not considered in the present paper are present and can extend the result
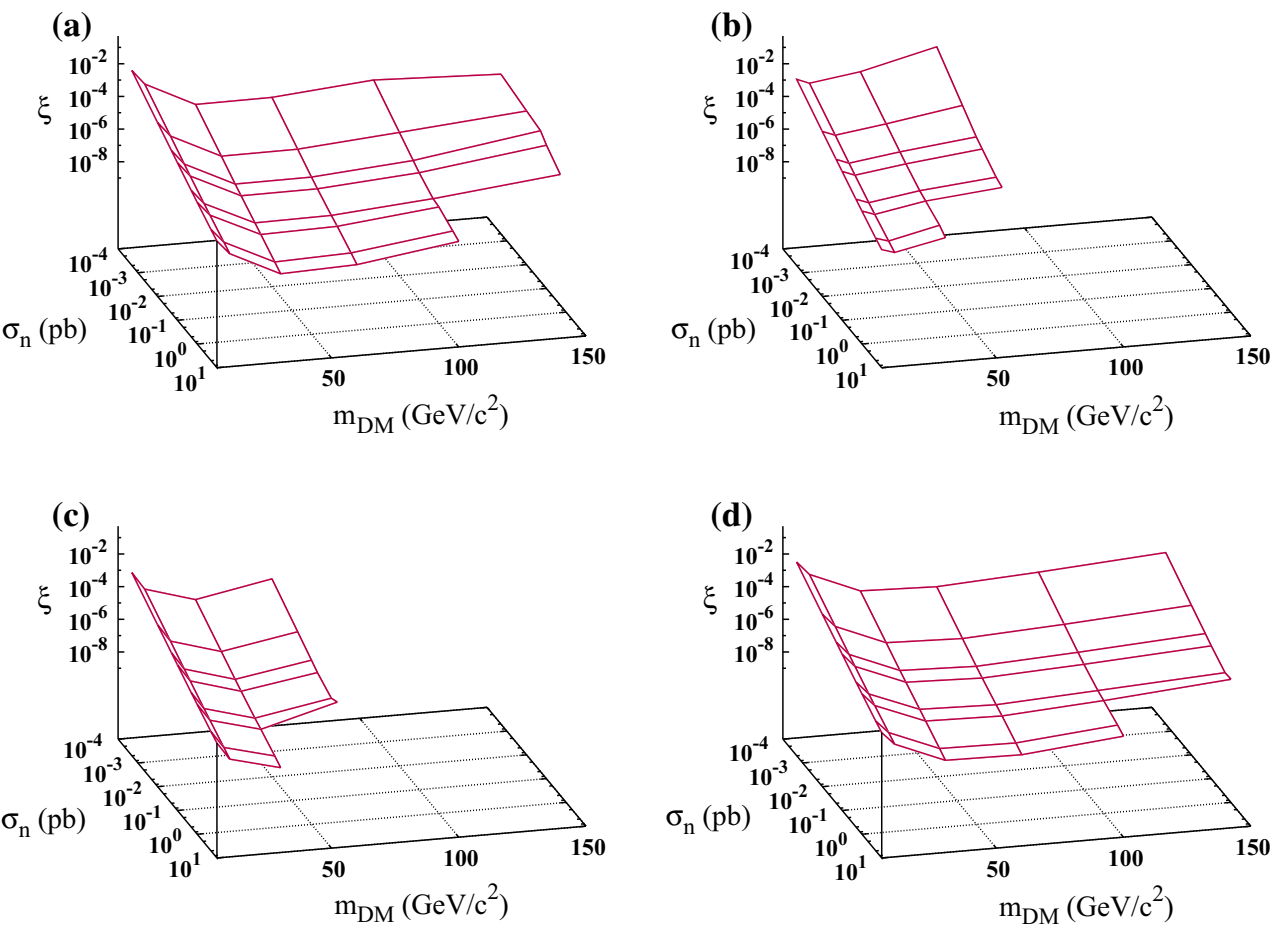

Fig. 8 Examples of the mean values of the allowed region of $\xi$ as function of $\sigma_{n}$ and $m_{D M}$, represented as an allowed surface (see text). The plots have been obtained for $v_{0}=220 \mathrm{~km} / \mathrm{s}$ in the same sets of parameters as in Fig. 7. See text 

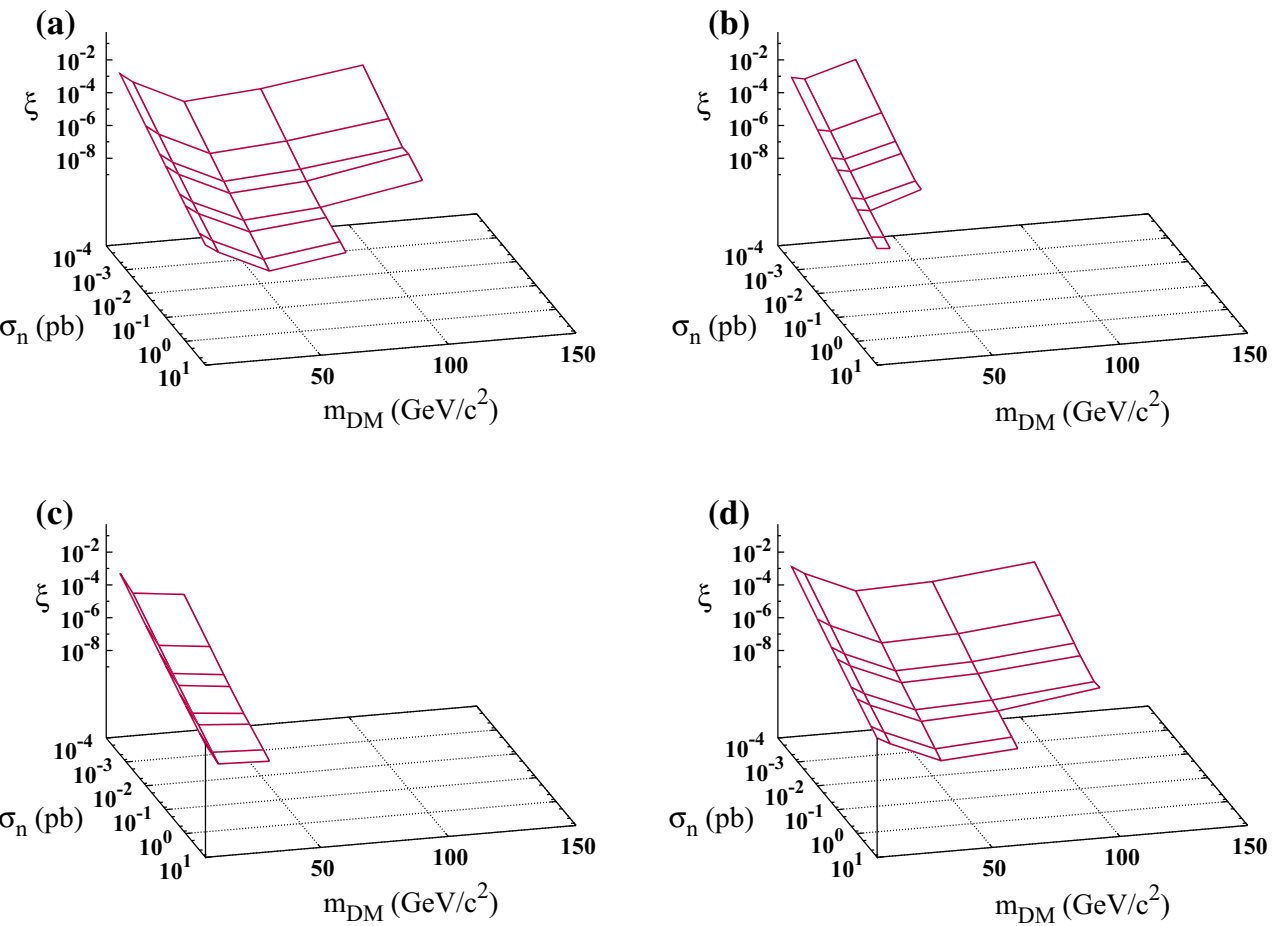

Fig. 9 Examples of the mean values of the allowed region of $\xi$ as function of $\sigma_{n}$ and $m_{D M}$, represented as an allowed surface (see text). The plots have been obtained for $v_{0}=270 \mathrm{~km} / \mathrm{s}$ in the same sets of parameters as in Fig. 7. See text

sidered scenario for quenching factors $\mathrm{Q}_{I}$ with channeling effect, B parameters set, $v_{0}=220 \mathrm{~km} / \mathrm{s}$ and $m_{D M}=60$ $\mathrm{GeV}$, the obtained upper limits on $\xi$ do exclude $\sigma_{n}>0.05$ $\mathrm{pb}$ and $\xi>10^{-3}$. When also including other uncertainties as other halo models etc. the results would be extended.

Open Access This article is distributed under the terms of the Creative Commons Attribution 4.0 International License (http://creativecomm ons.org/licenses/by/4.0/), which permits unrestricted use, distribution, and reproduction in any medium, provided you give appropriate credit to the original author(s) and the source, provide a link to the Creative Commons license, and indicate if changes were made. Funded by SCOAP $^{3}$.

\section{References}

1. R. Bernabei et al., Nucl. Instrum. Methods A 592, 297 (2008)

2. R. Bernabei et al., Eur. Phys. J. C 56, 333 (2008)

3. R. Bernabei et al., Eur. Phys. J. C 67, 39 (2010)

4. R. Bernabei et al., Eur. Phys. J. C 73, 2648 (2013)

5. P. Belli et al., Phys. Rev. D 84, 055014 (2011)

6. R. Bernabei et al., J. Instrum. 7, P03009 (2012)

7. R. Bernabei et al., Eur. Phys. J. C 72, 2064 (2012)

8. R. Bernabei et al., Int. J. Mod. Phys. A 28, 1330022 (2013)

9. R. Bernabei et al., Eur. Phys. J. C 62, 327 (2009)

10. R. Bernabei et al., Eur. Phys. J. C 72, 1920 (2012)

11. R. Bernabei et al., Eur. Phys. J. A 49, 64 (2013)

12. R. Bernabei et al., Eur. Phys. J. C 74, 2827 (2014)

13. R. Bernabei et al., Eur. Phys. J. C 74, 3196 (2014)
14. P. Belli, R. Bernabei, C. Bacci, A. Incicchitti, R. Marcovaldi, D. Prosperi, DAMA proposal to I.N.F.N. Scientific Committee II (1990)

15. R. Bernabei et al., Phys. Lett. B 389, 757 (1996)

16. R. Bernabei et al., Phys. Lett. B 424, 195 (1998)

17. R. Bernabei et al., Phys. Lett. B 450, 448 (1999)

18. P. Belli et al., Phys. Rev. D 61, 023512 (2000)

19. R. Bernabei et al., Phys. Lett. B 480, 23 (2000)

20. R. Bernabei et al., Phys. Lett. B 509, 197 (2001)

21. R. Bernabei et al., Eur. Phys. J. C 23, 61 (2002)

22. P. Belli et al., Phys. Rev. D 66, 043503 (2002)

23. R. Bernabei et al., Il Nuovo Cim. A 112, 545 (1999)

24. R. Bernabei et al., Eur. Phys. J. C 18, 283 (2000)

25. R. Bernabei et al., La Riv. del Nuovo Cim. 26(1), 1-73 (2003)

26. R. Bernabei et al., Int. J. Mod. Phys. D 13, 2127 (2004)

27. R. Bernabei et al., Int. J. Mod. Phys. A 21, 1445 (2006)

28. R. Bernabei et al., Eur. Phys. J. C 47, 263 (2006)

29. R. Bernabei et al., Int. J. Mod. Phys. A 22, 3155 (2007)

30. R. Bernabei et al., Eur. Phys. J. C 53, 205 (2008)

31. R. Bernabei et al., Phys. Rev. D 77, 023506 (2008)

32. R. Bernabei et al., Mod. Phys. Lett. A 23, 2125 (2008)

33. R. Bernabei et al., Phys. Lett. B 408, 439 (1997)

34. P. Belli et al., Phys. Lett. B 460, 236 (1999)

35. R. Bernabei et al., Phys. Rev. Lett. 83, 4918 (1999)

36. P. Belli et al., Phys. Rev. C 60, 065501 (1999)

37. R. Bernabei et al., Il Nuovo Cim. A 112, 1541 (1999)

38. R. Bernabei et al., Phys. Lett. B 515, 6 (2001)

39. F. Cappella et al., Eur. Phys. J. C 14, 1 (2002)

40. R. Bernabei et al., Eur. Phys. J. A 23, 7 (2005)

41. R. Bernabei et al., Eur. Phys. J. A 24, 51 (2005)

42. R. Bernabei et al., Astropart. Phys. 4, 45 (1995)

43. R. Bernabei, in The Identification of Dark Matter, ed. by N.J.C. Spooner (World Scientific Publication, Singapore, 1997), p. 574

44. K.A. Drukier et al., Phys. Rev. D 33, 3495 (1986) 
45. K. Freese et al., Phys. Rev. D 37, 3388 (1988)

46. J.I. Collar, F.T. Avignone III, Phys. Lett. B 275, 181 (1992)

47. J.I. Collar, F.T. Avignone III, Phys. Rev. D 47, 5238 (1993)

48. C. Kouvaris, I.M. Shoemaker, Phys. Rev. D 90, 095011 (2014)

49. G. Zaharijas, G.R. Farrar, Phys. Rev. D 72, 083502 (2005)

50. F. Hasenbalg et al., Phys. Rev. D 55, 7350 (1997)

51. G.D. Mack, J.F. Beacom, G. Bertone, Phys. Rev. D 76, 043523 (2007)

52. R. Foot, J. Cosmol. Astropart. Phys. 04, 014 (2012)

53. P.J.T. Leonard, S. Tremaine, Astrophys. J. 353, 486 (1990)

54. C.S. Kochanek, Astrophys. J. 457, 228 (1996)
55. K.M. Cudworth, Astron. J. 99, 590 (1990)

56. J. Delhaye, Stars and Stellar Systems, vol. 5 (Univ. of Chicago Press, Chicago, 1965) , p. 73

57. M.C. Smith et al., MNRAS 379, 755 (2007)

58. A.M. Dziewonski, D.L. Anderson, Phys. Earth Planet. Inter. 25, 297 (1981)

59. R.H. Helm, Phys. Rev. 104, 1466 (1956)

60. A. Bottino et al., Astropart. Phys. 2, 77 (1994)

61. V.I. Tretyak, Astropart. Phys. 33, 40 (2010)

62. S.I. Matyukhin, Tech. Phys. 53, 1578 (2008)

63. N. Bozorgnia et al., J. Cosmol. Astropart. Phys. 11, 19 (2010) 